\title{
Stromal osseous metaplasia in urothelial carcinoma of the bladder: a rare case report and literature review
}

\author{
Joëlle Razafimahefa ${ }^{1 *} \mathbb{D}$, Clément Gosset ${ }^{2}$, Pierre Mongiat-Artus ${ }^{3}$, Tsitohery Francine Andriamampionona ${ }^{1}$ and
} Jérôme Verine ${ }^{2,4,5}$

\begin{abstract}
Background: The bone formation within bladder tumors could be encountered in 3 conditions. These might consist of malignant bone formation in mesenchymal tumors; mixed mesenchymal and epithelial tumors; and epithelial tumors with stromal osseous metaplasia (SOM). This last is relatively rare. According to the English literature, only 12 cases have been reported in primary tumor and 7 in metastatic deposits of bladder primaries. Herein, we presented an additional case.

Case presentation: An 83-year-old man was admitted 13 years ago for prostatic adenocarcinoma, treated with radical prostatectomy. Biochemical recurrence was detected 2 years after surgery (prostate-specific-antigen (PSA) level: $4.60 \mathrm{ng} / \mathrm{mL}$ ) and progressively normalized $(<1.0 \mathrm{ng} / \mathrm{mL})$ after adjuvant radiotherapy and annual injection of leuprorelin (enantone ${ }^{\mathrm{R}}$ ). He was referred after 8 years for hematuria, PSA level having slightly increased $(0.60 \mathrm{ng} / \mathrm{ml})$. Cystoscopy showed a nodular growth in the bladder wall, visualized as a calcified tumor on computed tomography $(\mathrm{CT})$ and removed with transurethral resection. Histologically, the tumor consists of a non-muscle-invasive high grade papillary urothelial carcinoma with metaplastic bone within the stroma. Immunohistochemical analysis particularly demonstrated positive expression of respectively CD56 on osteoblasts, and CD68 on osteoclasts. MDM2 and CDK4 were negatives on osteoid and bone tissue. Six courses of Bacillus Calmette-Guerin (BCG) therapy have been administered. Two local recidives have occurred during an 8-month follow-up period after immunotherapy and were treated with six further courses of BCG therapy. At one-month follow-up, the patient was well without remaining symptoms.
\end{abstract}

Conclusion: SOM is a rare benign condition whose pathogenesis remains uncompletely defined. Sarcomatoïd carcinoma represents the main differential diagnosis that influences therapeutic procedures. Prognosis depends essentially on the extent of the carcinomatous component .

Keywords: Stromal osseous metaplasia, Metaplastic bone, Osteoid, Urothelial carcinoma, Bladder, Alkaline phosphatase

\section{Background}

The bone formation within bladder tumors, isolated or associated with cartilage formation, can be schematically divided into 3 distinct groups [1-4]. The first group consists of malignant bone formation in mesenchymal tumors (osteosarcoma). The second group corresponds to sarcomatoid urothelial carcinomas where the bone formation is

\footnotetext{
* Correspondence: rootsrazaf@yahoo.fr

${ }^{1}$ UPFR Anatomie et Cytologie Pathologiques, CHU, Andrainjato, Fianarantsoa, Madagascar

Full list of author information is available at the end of the article
}

considered as an heterotopic sarcomatous element associated with a carcinomatous component $[1,4]$. In the third group, the bone formation appears as a stromal osseous metaplasia, that is, the transformation of a part of the tumoral stroma of urothelial carcinomas into mature bone, characterized by reactive benign bone trabeculae forming a lamellar architecture [3]. This last condition is rarely encountered in bladder tumors and represents a curious finding, firstly described in urothelial carcinoma by Pang et al. in 1958 [1]. Since then, a total of 19 cases have been reported in urothelial carcinoma among which 12 cases 
were found in primary tumors and 7 in metastatic locations [2].

We reported herein an additional case of stromal osseous metaplasia in a high grade papillary urothelial carcinoma of the urinary bladder.

\section{Case presentation}

An 83-year-old man was firstly admitted to hospital 13 years ago for prostatic adenocarcinoma and underwent radical prostatectomy. He also received adjuvant radiotherapy and annual injection of leuprorelin (enantone ${ }^{\mathrm{R}}$ ) for a biochemical recurrence characterized by a prostatespecific-antigen (PSA) level of $4.60 \mathrm{ng} / \mathrm{mL}$ detected 2 years after surgery. Clinical evolution was favorable and serum PSA level has progressively decreased to lesser than 0.1 $\mathrm{ng} / \mathrm{mL}$. At 8 -years follow-up, he presented with an abundant gross hematuria. Serum PSA level was slightly increased $(0.60 \mathrm{ng} / \mathrm{ml})$. A cystoscopy was performed showing a nodular tumor growth on the right side of the lateral bladder wall that appeared as a large calcified tumor on computed tomography. Transurethral resection of bladder (TURB) was carried out. Tumor specimens consisted of small fragments of whitish firmer tissue. Microscopic examination showed frond like papillary projections which were lined by thick layers of stratified transitional epithelial cells with central fibrovascular cores. There was a predominant disorderly pattern with marked variation of architectural features. Tumor epithelial cells focally showed prominent cytologic abnormalities with moderate to significant nuclear pleomorphism. Quite numerous mitotic figures were observed and prominent nucleoli were also present in places. In some areas, the tumor cells disrupted the basement membrane and invaded into the lamina propria. The muscularis propria was respected. All these findings were consistent with the diagnosis of high-grade papillary urothelial carcinoma with infiltration of lamina propria (pT1 stage). Coexisting urothelial carcinoma in situ was found focally (Cis). The tumor stroma contained multiple bone trabeculae of variable size, lamellated or coarsed-fibred and separated by loose fibrous connective tissue with osteoid seams (Fig. 1a). Those osteoid and bony structures were outlined by numerous isolated large cells with abundant blue cytoplasm and quite numerous multinucleated giant cells that respectively correspond to osteoblasts and osteoclasts, without atypia nor pleomorphic stromal cells (Fig. 1b). No cartilage formation was observed. Immunohistochemistry showed in osteoid areas and bone tissue, a positive expression of CD56 (Fig. 1c) in osteoblasts. There was a discrete and heterogeneous staining for PS100, a discrete immunoreactivity for P53, and a Ki67 index of $20 \%$ in tumor cells. MDM2 and CDK4 immunostaining was negative in both metaplastic bone and carcinomatous components. Multinucleated osteoclasts were also strongly stained by CD68 (Fig. 1d). At 2-months follow-up after TURB, a control cystoscopy was performed and showed necrotic zone and

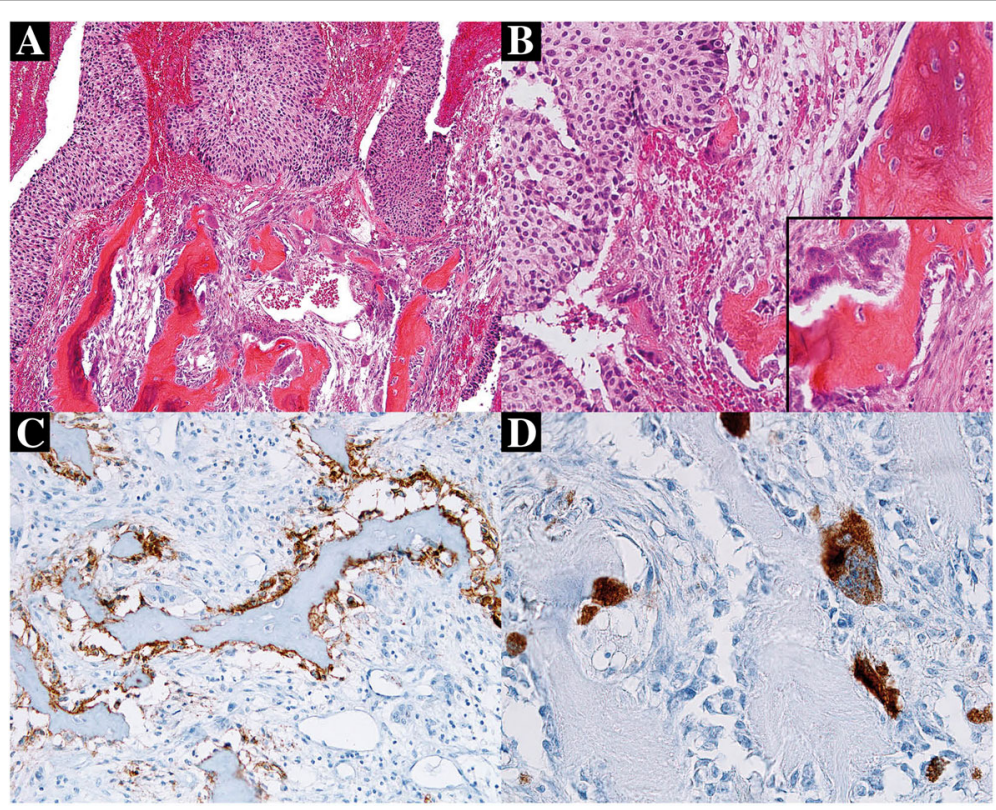

Fig. 1 Stromal osseous metaplasia in urothelial carcinoma of the bladder. a Tumor stroma containing multiple bone trabeculae of variable size, separated by loose fibrous connective tissue with osteoid seams (Hematoxylin and Eosin Stain $\times 200$ ). b Osteoid and bone trabeculae outlined by large cells with abundant cytoplasm and multinucleated giant cells, corresponding respectively to osteoblasts and osteoclasts. Transitional cell carcinomatous component is seen on the left. (Hematoxylin and Eosin Stain $\times 400$ ). c Positive and diffuse membrane staining of osteoblasts with CD56 antibody. $\mathbf{d}$ Strong positive cytoplasmic staining pattern of osteoclasts with CD68 antibody 
retractile fibrous scar on the right side of the bladder trigone which were completely excised by secondary TURB. Those remaining lesions correspond essentially to fibroinflammatory changes without residual tumor. Six courses of BCG therapy have been indicated. Two local recidives with recurrent episodes of gross hematuria have occurred during an 8-month follow-up period after immunotherapy. These local tumor recurrences displayed the same histological findings that demonstrated a high grade papillary urothelial carcinoma with superficial invasion of the lamina propria but were devoided of SOM foci. The multidisciplinary discussion recommended six further courses of BCG therapy at the time of the first recurrence. The second tumor recurrence appeared 2 months later and has been completely excised.

At one-month follow-up, the patient was well and did not complain of any symptoms.

\section{Discussion}

Stromal osseous metaplasia is an unusual entity, rarely encountered in urothelial bladder carcinomas. There are 19 reports of such cases recorded to date in the English literature. These are summarized with the present case in Table 1 and Table 2. Patients are predominantly males (male to female ratio: 3.25 ) with a median age of 65 years (range 3 to 85 ). There was a unique pediatric case observed in a 3-year-old boy [5].

Among these cases, 12 occurred in primary tumor without predilection for any particular site within the bladder. In almost half of those cases, a past history of partial cystectomy or radical prostatectomy have been noticed 5 months to 7 years before, eventually associated with repeated procedures of TURB. For the remaining 6 cases, there was no data available describing circumstances related to the primary tumor. In our case, SOM was detected in a primary bladder tumor that occurred 13 years after a prostatic adenocarcinoma, treated by radical prostatectomy with adjuvant radiotherapy, followed by annual injection of leuprorelin (enantone ${ }^{\mathrm{R}}$ ).

Moreover, apart from those 12 previous cases of primary urothelial carcinoma, 4 other cases of bladder carcinoma including one squamous, two undifferentiated and one adenocarcinoma have been reported by Eble and Young [2] in 1991 as associated with SOM. Besides, it is noteworthy that moderate to significant mucin deposits have been observed within the two latter variants.

Osseous metaplasia has been also found associated with metastatic tumors in 7 cases. Bone represents the most common site of metastasis and tibia is mainly involved associated as well with femur involvement [12, 13]. In such cases, radiographic evidence of new bone deposits has been noticed and heterotopic ossification has been described in soft tissue adjacent to the metastatic urothelial carcinoma. Other localizations including regional lymph nodes [3, 14], supraclavicular region [12], retroperitoneal space [3] and abdominal wall [11] have been also encountered.

Although the prognosis is essentially determined by the extent of the urothelial carcinoma, the metaplastic bone component could sometimes be increased considerably in size and thus required imaging procedures for follow-up. These included computed tomography (CT), magnetic resonance imaging (MRI) and positron emission tomography computed tomography (PET-CT). This has been illustrated in a case of retroperitoneal metastasis reported by Boudabbous et al., through which CT has been demonstrated as the best imaging modality that allows follow-up of lesion and detection of new lesions. CT imaging feature consists of hyperdense mass resembling cortical bone that shows moderate hypermetabolism (maximum SUV, 5.1) on PET - CT and appears hypointense on all sequences with peripheral enhancement on MRI.

The mechanism and the pathogenesis of metaplastic bone have not been completely elucidated [15]. However, we can firstly note that the urothelial carcinoma was of high grade (Grade 3) in the great majority of the 20 cases described with SOM, including our case. Grade 2 and grade 1 have been only reported respectively in 4 cases $[2,4,6]$ and 2 cases $[1,11]$. Tumor grade was not available in the last 4 cases $[8,13]$. A possible relationship between the tumor grade and the bone forming capacity of urothelial cells could be evocated, suggesting that this capacity would be enhanced when the grade increased. Moreover, some authors suggested a possible correlation between the bladder epithelium and the local concentration of calcium, inorganic phosphate as well as the alkaline-phosphatase activity [1]. They revealed through several experiments the ability of urinary epithelium to induce osseous metaplasia. This is not due to its cell specificity but to its chemical and enzymatic products and also to its property to keep local enzymatic concentration constantly high, the main condition to obtain metaplastic bone [1,3]. Another hypothesis suggested a transformation of stromal mesenchymal cells into osteoblasts which could be induced by bone morphogenetic proteins (BMP). These last correspond to multi-functional growth factors that belong to the transforming growth factor $\beta$ (TGF $\beta$ ) superfamily which play especially an important role in new bone formation. In particular BMP 2 and BMP $1 \mathrm{~b}$ are considered as active inducers of osteoblastic metaplasia respectively in primary and metastatic tumor sites $[1,3]$. The fact that primary tumors in reported cases were preceded by surgery or TURB suggests the presence of scar tissue and fibrous change in which there would be an increased alkaline-phosphatase activity and fibroblasts would be more susceptible to bone evoking agents, particularly to BMP leading to bone formation. This hypothesis could 
Razafimahefa et al. Diagnostic Pathology

(2019) $14: 75$

Page 4 of 9

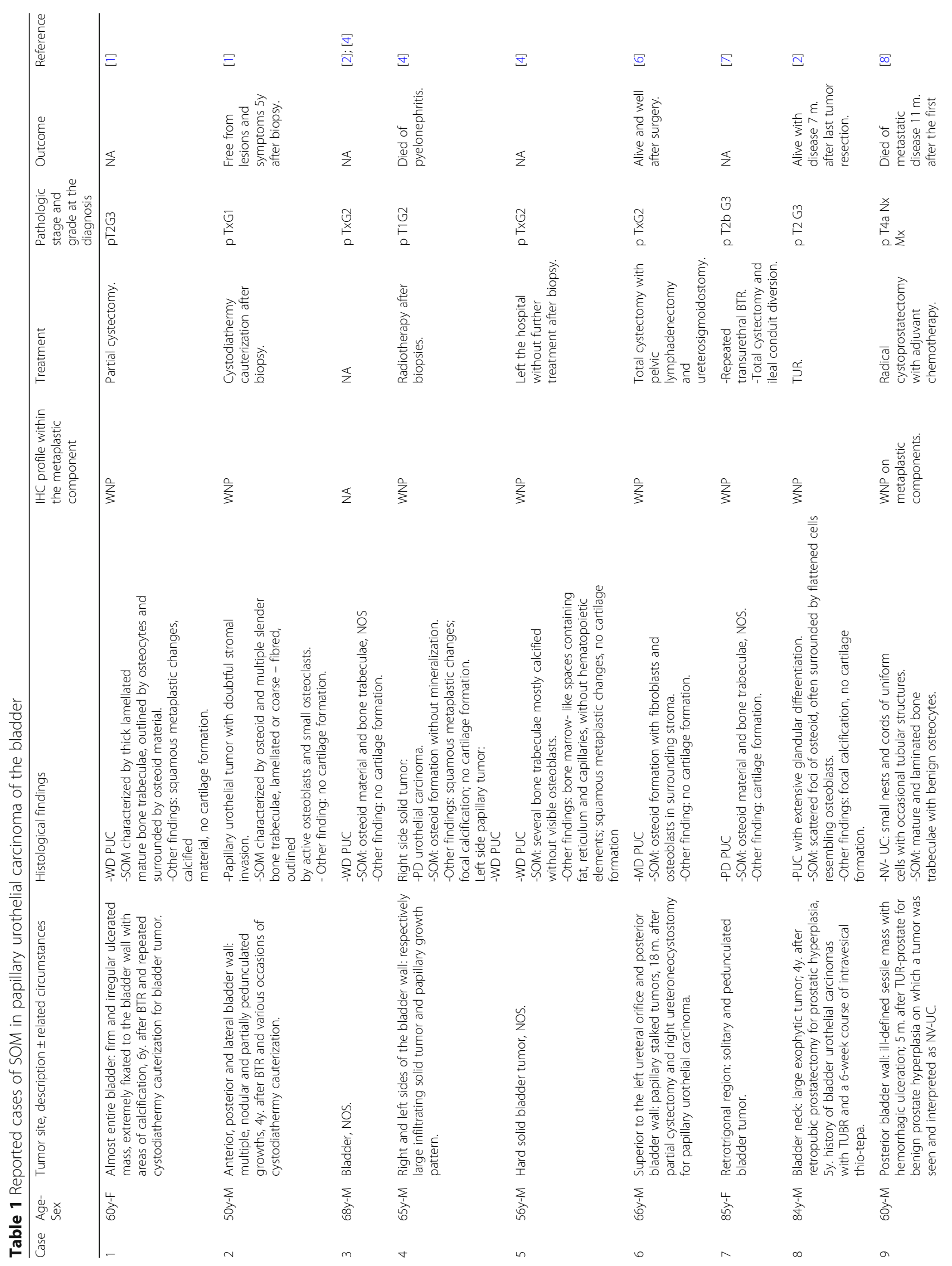


Razafimahefa et al. Diagnostic Pathology

(2019) $14: 75$

Page 5 of 9

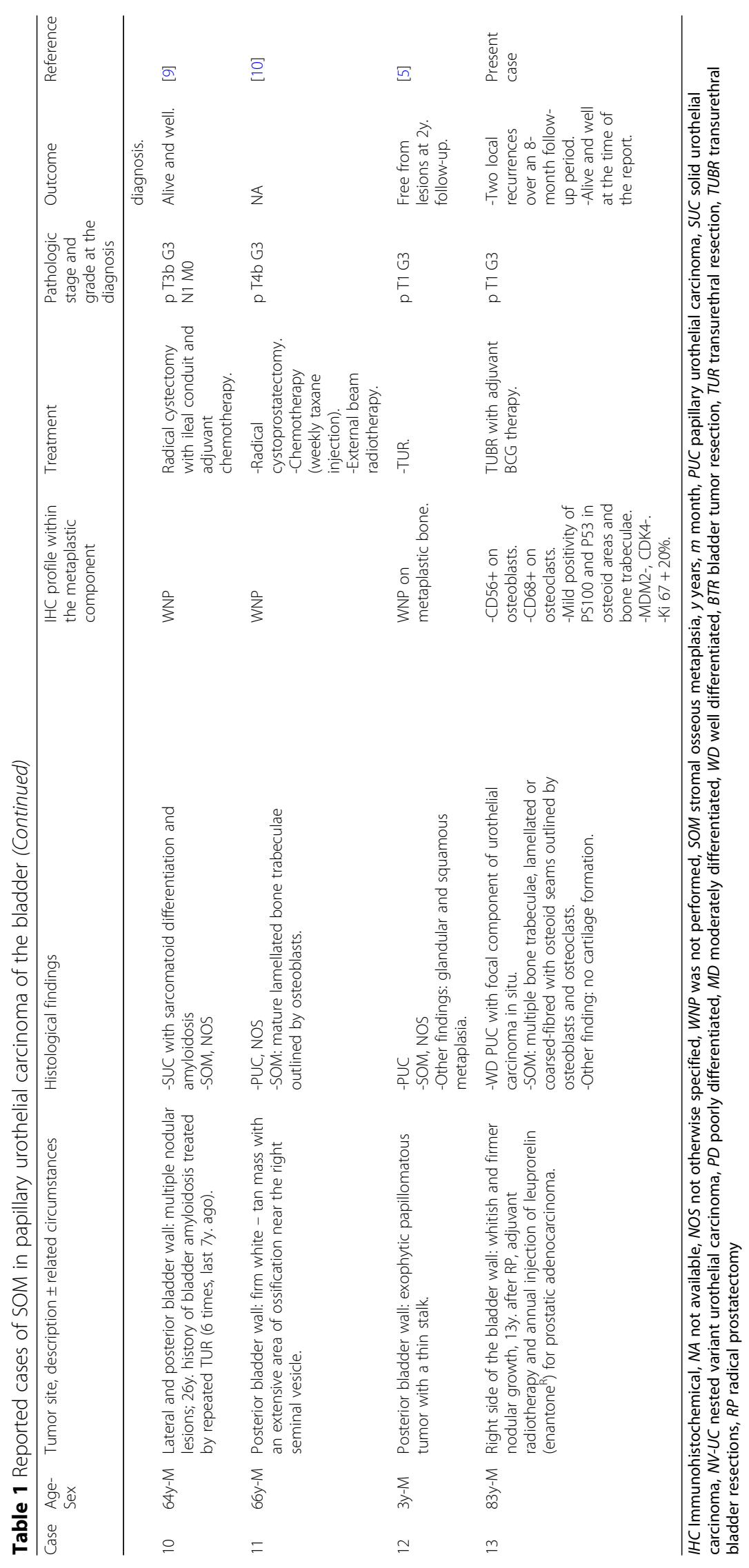




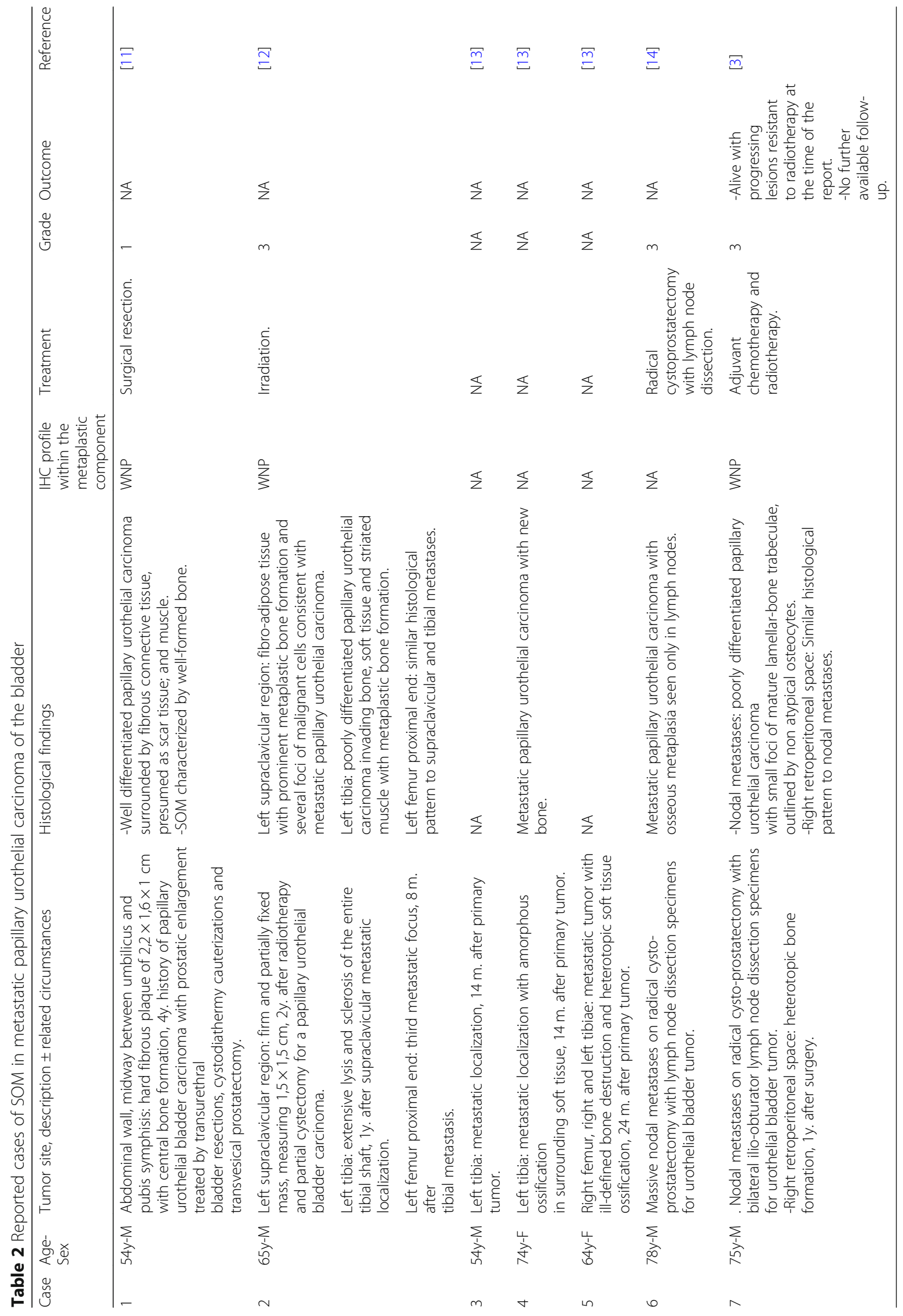


be also considered in our case toward the history of radical prostatectomy and adjuvant radiotherapy. In addition, annual leuprorelin injection which is a luteinizing hormone releasing hormone agonist (LHRHa) plays an important role in normal skeleton homeostasis [16]. Leuprorelin therapy would in fact decrease bone mineral density and increase local concentration of alkaline phosphatase that could contribute to metaplastic bone formation [16, 17].

Some authors have also attempted to suggest that extracellular mucin deposition contributes to maintain an alkaline zone which have been found necessary for optimum activity of alkaline phosphatase. Furthermore, other factors of osteogenesis that encompass specific transcription factors such as Oct $3 / 4$, Sox 2 , c-Myc and Klf4 have been described. These lasts have been found to generate pluripotent stem cells. Under the influence of these similar transcription factors, fibroblasts that are present within the stromal component could differentiate into osteoblasts [18]. The pathogenesis of SOM associated with non-urothelial bladder carcinoma could be thereby correlated to bone inducing properties of extracellular mucin and specific transcription factors, as well as the bone evoking activity of the urothelium.

Therefore, 3 conditions would be necessary for bone formation which include osteogenic precursor cells, inducing agents and a permissive environment. A genetic predisposition may also be implicated and could explain the only pediatric case of SOM within a high grade papillary urothelial carcinoma despite the absence of particular triggering factors.

More interestingly, all those hypotheses could be comforted by the fact that SOM were also encountered in metastatic bladder tumor that involved regional lymph nodes, bones and tissue in close proximity, i.e. supraclavicular region, abdominal wall and retroperitoneal space within the context of previous surgery. As metastatic sites occurred in bone or proximities as well as in tissue scar, this would suggest a high local concentration of bone-evoking agents which favors SOM.

Osseous metaplasia should be recognized as a nonneoplastic change that could be encountered in benign (inflammatory lesions, benign tumors) and malignant conditions as seen in our present case, given that urothelial cells are wellknown as bone-inducing agent $[1,2]$. Microscopically, benign metaplastic bone usually consists of irregular, mature and lamellated bone trabeculae, outlined by osteoblasts, osteocytes and eventual osteoclasts. Those cells do not demonstrate atypia and are relatively distinct from the mesenchymal cells of the adjacent stroma. Osteoid deposits are also found and often areas of hemorrhage are present [19]. The routine use of immunohistochemistry is not mandatory to assess the diagnosis as morphological features are usually well-defined. This could explain the absence of immunohistochemical (IHC) analysis for SOM in all those cases described. However, specific antibody stains could be performed. Osteoblasts stain positive for alkaline phosphatase, CD56 and SATB2. Expression pattern of osteonectin and osteocalcin could be found on osteoblasts, as well as on osteocytes and the surrounding matrix. Osteoclasts demonstrate positive immunoreactivity for Tartrate-resistant acid phosphatase (TRAP) and CD68. Special stains such as von Kossa, Masson's and Goldner's Trichrome could be also used to differentiate osteoid deposits [20].

The main histological differential diagnoses that should be distinguished from SOM in urothelial bladder carcinoma are sarcomatoid carcinomas (SC) of the bladder which constitute an important challenge for they definitely change the course and the treatment of the case $[1,4]$. In the last World Health Organization classification of urinary tract tumors, these lesions are defined as biphasic neoplasms composed of an admixture of malignant epithelial and mesenchymal elements [21, 22]. These lasts could demonstrate osteosarcomatous differentiation, usually formed by urothelial carcinoma coexisting with osteosarcoma in which bone formation is an element of the mesenchymal malignant component. This variant of SC should be firstly eliminated, even though the incidence is rare. The mesenchymal component is characterized by high grade sarcoma with spindle, oval or round tumor cells of variable size showing marked nuclear pleomorphism. Bizarre giant cells and osteoclast-like multinucleated giant cells are not uncommon. Those cells are usually seen within abundant lace-like osteoid that could be associated with chondroid matrix. MDM2 and CDK4 immunostaining are not specific for diagnosis but could be expressed by tumor cells. Osteoid matrix stains positive for vimentine and alkaline phosphatase and positive immunostaining for S-100 protein could be identified in chondroid areas. Ki 67 and p53 are not specific but could be considered as indicators of malignant potential. They are highly expressed in osteosarcoma and usually negative or mildly expressed in metaplastic conditions. This histological variant of urothelial carcinoma has a poor prognosis. The treatment consists of radical cystectomy [15, 23]. Tumor cells are occasionally radio-chemosensitives. Most patients die within 6 months due to local spread with urinary tract obstruction and secondary infection [23].

Noteworthily, in this context of SC, urothelial carcinoma could also coexist with leiomyosarcoma, chondrosarcoma, rhabdomyosarcoma, rarely liposarcoma, and more than one type of heterologous differentiation may be present [15]. Those components are obviously morphologically different from metaplastic bone and thus, should not be specifically considered as diagnoses problems.

In the context of extensive metaplastic bone as well as metastatic urothelial carcinoma associated with SOM, differential diagnosis of bone formation should be also 
established on imaging. Radiation-induced osteosarcoma should be firstly suspected in patients who have undergone radiotherapy, as far as lesions involved the irradiation field. A latency of 6 months from the exposure is sufficient to affirm the diagnosis. Secondly, extraskeletal osteosarcoma should also be evocated. Patients are mostly adults, aged over 40 years and the prognosis is almost always poor. The typical CT presentation of osteosarcoma consists of heterogeneous areas with focal hypoattenuation that corresponds to necrosis or hemorrhage. On MRI, these tumors usually demonstrate intermediate signal on T1 with enhancement or low signal both in T1 and T2 sequences. Sarcomatoid carcinoma, recognized as the main differential diagnosis of urothelial bladder carcinoma with SOM, should be also suspected on imaging, especially on CT and consists of a large heterogeneous mass with hyperdense components.

The presence of metaplastic bone within bladder urothelial carcinoma does not change the therapeutic process that essentially differs according to tumor stage and depends on muscular invasion [24, 25].

Of the 12 cases of primitive tumors reported with SOM, 4 cases had favorable outcomes $[1,5,6,9]$. In these cases, patients were correctly treated using procedures that are based on consensus guidelines according to tumor stages. Three other cases had negative outcomes characterized by patient death $[4,8]$ or persistent lesion on follow-up [2]. Death is usually due to metastasis or unresectable tumors with or without associated complications such as pyelonephritis $[2,4,8]$. In the 5 remaining cases, outcomes were not available $[1,2,4,7$, 10]. Concerning the 7 cases of metastatic urothelial carcinoma reported in the literature, only one case [3] had available outcomes, marked with disease progression mainly resistant to radiotherapy.

We could notice through those observed clinical courses, including the present case, that the disease outcomes are based on treatment, particularly on its response or effectiveness as well as on its clinical feasibility. The quality of follow-up may also have a major impact on outcomes and survival.

\section{Conclusion}

Stromal osseous metaplasia is a benign condition rarely found within urothelial bladder carcinoma. Detailed mechanisms underlying this phenomenon have not been completely elucidated and need further investigations. The presence of this feature does not change the treatment approach of urothelial carcinoma which is related to histologic grade and tumor stage. This last is relatively based on muscle invasion. However, metaplastic bone should be differentiated from malignant osteogenous conditions. Sarcomatoid carcinoma constitutes the main differential diagnosis that obviously changes the treatment approach and thus represents a challenging problem. The prognosis is poor in all cases of sarcomatoid carcinoma and outcomes are characterized by a high incidence of recurrences. Therefore, a long-term follow-up is recommended.

\section{Abbreviations \\ BCG: Bacillus Calmette-Guerin; BMP: Bone morphogenetic proteins; \\ CT: Computed tomography; IHC analysis: Immunohistochemical analysis; LHRHa: Luteinizing hormone releasing hormone agonist; MRI: Magnetic resonance imaging; PET-CT: Positron emission tomography computed tomography; PSA: Prostate-specific-antigen; SC: Sarcomatoïd carcinoma; SOM: Stromal osseous metaplasia; TGFß: Transforming growth factor B; TURB: Transurethral resection of bladder}

\section{Acknowledgements}

Not applicable.

Authors' contributions

All the authors contributed to the acquisition of data, revised the paper and gave final approval.

\section{Funding}

The authors declare that this paper was not financially supported.

Availability of data and materials

All data generated or analyzed during this study are included in this published article.

Ethics approval and consent to participate

Not applicable.

\section{Consent for publication}

Written informed consent was obtained from the patient for publication of this Case Report and any accompanying images. A copy of the written consent is available for review by the Editor of this journal.

\section{Competing interests}

The authors declare that they have no competing interests.

\section{Author details}

'UPFR Anatomie et Cytologie Pathologiques, CHU, Andrainjato, Fianarantsoa, Madagascar. ${ }^{2}$ Service de Pathologie, Hôpital Saint-Louis, AP-HP, Paris, France. ${ }^{3}$ Service d'Urologie, Hôpital Saint-Louis, AP-HP, Paris, France. ${ }^{4}$ Service de Recherches en Hémato-immunologie, CEA, Hôpital Saint-Louis, Paris, France.

${ }^{5}$ Université Paris Diderot, Institut Saint - Louis, Paris, France.

Received: 4 January 2019 Accepted: 26 June 2019

Published online: 13 July 2019

References

1. Shu-Chao P, Pang LS. Bony and cartilaginous tumours of the urinary bladder. J Pathol. 1958;76(2):357-77.

2. Eble JN, Young RH. Stromal osseous metaplasia in carcinoma of the bladder. J Urol. 1991;145(4):823-5.

3. Boudabbous S, Arditi D, Paulin E, Koessler T, Rougemont AL, Montet X. Ossifying metaplasia of urothelial metastases: original case with review of the literature. BMC Med Imaging. 2015;15(1):30.

4. Delides GS. Bone and cartilage in malignant tumours of the urinary bladder. BJU Int. 1972:44(5):571-81.

5. Kral M, Michalek J, Skarda J, Tichy T, Smakal O, Kodet R, Student V. Highgrade urothelial bladder cancer in children: A case report and critical analysis of the literature. Biomed Pap Med Fac Univ Palacky Olomouc Czech Repub. 2016;160(4):578-82.

6. Toma H, Yamashita N, Nakazawa H, Yamaguchi Y. Transitional cell carcinoma with osteoid metaplasia. Urology. 1986;27(2):174-6.

7. Nakachi K, Miyamoto I, Kuroda J, Kohro T. A case of transitional cell carcinoma of the bladder with heterotopic bone formation. Hinyokika kiyo. Acta urologica Japonica. 1988;34(9):1651-5. 
8. Neşe N, Kandiloğlu AR, Atesçi YZ. Nested variant of transitional cell carcinoma with osseous metaplasia of the urinary bladder: a case report and review of published reports. International journal of urology. 2007;14(4): $365-7$.

9. Gupta P, Hanamshetti S, Kulkarni JN. Primary amyloidosis with high grade transitional cell carcinoma of bladder: a rare case report. J Cancer Res Ther. 2012;8(2):297.

10. Dudley AG, Tomaszewski JJ, Hughes AH, Davies BJ. Incidentally discovered osseous metaplasia within high-grade urothelial carcinoma of the bladder. Urology. 2012;79(4):e59-60.

11. Cornes JS, Sussman T, Dawson IM. Bone formation in metastasis from carcinoma of urinary bladder: report of a case with review of the literature. BJU International. 1960;32(3):290-4

12. Chinn DA, Genant HK, Quivey JM, Carlsson AM. Heterotopic-bone formation in metastatic tumor from transitional-cell carcinoma of the urinary bladder. A case report. JBJS. 1976;58(6):881-3.

13. Evison G, Pizey N, Roylance J. Bone formation associated with osseous metastases from bladder carcinoma. Cli Radiol. 1981;32(3):303-9.

14. Cormio L, Sanguedolce F, Massenio P, Di GF, Selvaggio O, Bufo P, Carrieri G. Osseous metaplasia within a urothelial bladder cancer nodal metastasis: a case report. Anal Quant Cytopathol and Histopathol. 2014;36(2):117-9.

15. Tsoucalas G, Fiska A. Osseous Metaplasia of the Urinary Bladder: mini review of the cases reported after 1991. J Univer Surg. 2018;6(1):3.

16. Warde N. Prostate cancer: Degarelix controls serum alkaline phosphatase better than leuprolide. Nature Rev Urol. 2010;7(8):416.

17. Golub EE, Boesze-Battaglia K. The role of alkaline phosphatase in mineralization. Curr Opin Orthop. 2007;18(5):444-8.

18. Takahashi K, Tanabe K, Ohnuki M, et al. Induction of pluripotent stem cells from adult human fibroblasts by defined factors. Cell. 2007;131:861-72.

19. Bossche LV, Vanderstraeten G. Heterotopic ossification: a review. J Rehabil Med. 2005;37(3):129-36.

20. Hao Z, Kalscheur VL, Muir P. Decalcification of bone for histochemistry and immunohistochemistry procedures. J Histotechnol. 2002;25(1):33-7.

21. Moch H, Cubilla AL, Humphrey PA, Reuter VE, Ulbright TM. The 2016 WHO classification of tumours of the urinary system and male genital organs_-part A: renal, penile, and testicular tumours. European Urol. 2016; 70(1):93-105.

22. Humphrey PA, Moch H, Cubilla AL, Ulbright TM, Reuter VE. The 2016 WHO classification of tumours of the urinary system and male genital organs_-part B: prostate and bladder tumours. European Urol. 2016;70(1): 106-119.

23. Mittal V, Rupala KK, Yadav R, Suryavanshi M. Giant Sarcomatoid Carcinoma with Osseous Metaplasia from Urinary Bladder Diverticulum. Indian J Surg Oncol. 2017:8(3):436-9.

24. Babjuk M, Böhle A, Burger M, Capoun O, Cohen D, Compérat EM, Hernández V, Kaasinen E, Palou J, Rouprêt M, van Rhijn BW. EAU guidelines on non-muscle-invasive urothelial carcinoma of the bladder: update 2016. European Urol. 2017:71(3):447-61.

25. Stenzl A, Cowan NC, De Santis M, Jakse G, Kuczyk MA, Merseburger AS, Ribal MJ, Sherif A, Witjes JA. The updated EAU guidelines on muscleinvasive and metastatic bladder cancer. European Urol. 2009;55(4):815-25.

\section{Publisher's Note}

Springer Nature remains neutral with regard to jurisdictional claims in published maps and institutional affiliations.

Ready to submit your research? Choose BMC and benefit from:

- fast, convenient online submission

- thorough peer review by experienced researchers in your field

- rapid publication on acceptance

- support for research data, including large and complex data types

- gold Open Access which fosters wider collaboration and increased citations

- maximum visibility for your research: over $100 \mathrm{M}$ website views per year

At BMC, research is always in progress.

Learn more biomedcentral.com/submissions 